\title{
Extended Reach of 116 Gb/s DP-QPSK Transmission using Random DFB Fiber Laser Based Raman Amplification and Bidirectional Second-order Pumping
}

\author{
M. Tan, P. Rosa, I. D. Phillips, and P. Harper \\ Aston Institute of Photonic Technologies, Aston University, Birmingham B4 7ET, UK \\ tanm@aston.ac.uk
}

\begin{abstract}
We propose a novel random DFB fiber laser based Raman amplification using bidirectional second-order pumping. This extends the reach of $116 \mathrm{~Gb} / \mathrm{s}$ DP-QPSK WDM transmission up to $7915 \mathrm{~km}$, compared with other Raman amplification techniques.

OCIS codes: (060.1660) Coherent communications ; $(060.2320)$ Fibre optics amplifiers and osicllators;
\end{abstract}

\section{Introduction}

It is well known that using Raman amplification to provide distributed amplification can give a transmission performance improvement compared with lumped amplification. To minimize the generation of noise the distributed amplification would exactly counteract the fiber attenuation along the length of the transmission path, maintaining the signal power level at a near constant value [1]. In addition to minimizing noise, recent work has shown that a constant and/or symmetric power level is advantageous for some techniques used to compensate for nonlinear transmission effects $[2,3]$.

We have previously reported a technique which can achieve very low variation in signal power along the length of transmission fiber using an ultra-long Raman fiber laser (URFL) configuration with second-order Raman pump and fiber Bragg grating (FBG) based Fabry-Perot cavity [1]. This technique can give an almost negligible $1.7 \mathrm{~dB}$ signal power variation (SPV) over an $80 \mathrm{~km}$ fiber span. However this requires bidirectional pumping with equal forward and backward powers to minimize the SPV and noise. Unfortunately in transmission experiment the use of forward pumping is problematic as the penalty associated with relative intensity noise (RIN) transfer to the signal is greater than the performance improvement from low SPV \& noise [4]. Using only backward pumping improves the performance but at the expense of an increase in SPV and noise.

In this paper we report new Raman amplification configurations based on random distributed feedback (DFB) fiber laser and compare them with conventional Raman pumping and Fabry-Perot cavity based ultra-long Raman laser schemes. For 10 x $116 \mathrm{G}$ DP-QPSK WDM transmission we demonstrate a bidirectional, second-order Raman pumped random DFB fiber laser based amplification which simultaneously achieves low SPV and improved transmission performance. We show an extended reach of $7915 \mathrm{~km}$ using the proposed random laser based scheme with an SPV of $3.6 \mathrm{~dB}$, compared to $4999 \mathrm{~km}$ and $9 \mathrm{~dB}$ using backward first-order Raman pumping, and $7082 \mathrm{~km}$ using other amplification schemes. We also show a random laser with backward pumping configuration which uses only one pump but has performance comparable to conventional, dual-order (two pumps), backward pumping. In common with other random laser configurations, this scheme is easy to implement and has the potential to be insensitive to temperature variations [5].

\section{Experimental setup and characterizations of different Raman amplification techniques}

To evaluate the transmission performance, a recirculating loop experiment was conducted using the set-up shown in Fig. 1.(a). Ten DFB lasers with $100 \mathrm{GHz}$ spacing ranging from $1542.14 \mathrm{~nm}$ to $1549.32 \mathrm{~nm}$ were combined with a $100 \mathrm{kHz}$ linewidth tunable laser used as a "channel under test" through a polarization maintaining (PM) coupler while the corresponding DFB laser was switched off. The combined signals were QPSK modulated at 29 Gbaud. Normal and inverse $2^{31}-1$ PRBS patterns were used for I \& $Q$ with a relative delay of 18 bits. A PM EDFA was used to amplify the signal. The resultant $10 \times 116 \mathrm{~Gb} / \mathrm{s}$ DP-QPSK signals were generated by a polarization multiplexer with a 290-symbol delay between the two polarizations states before launching into the recirculating loop. The transmission span in the recirculating loop was $83.32 \mathrm{~km}$ standard SMF-28 fiber. The total loss was $\sim 17.6 \mathrm{~dB}$, including $\sim 16.5 \mathrm{~dB}$ from the fiber and $\sim 1.1 \mathrm{~dB}$ from 1366/1455/1550 WDMs. To equalize channel powers, a gain flattening filter (GFF) was used after the Raman link. The $\sim 12 \mathrm{~dB}$ loss from the GFF, 50/50 coupler, acousto-optic modulator (AOM), and Raman components was compensated using a single stage EDFA at the end of the loop. The output signal was demultiplexed by a tunable filter and amplified by an EDFA before the receiver. The receiver was a standard dual polarization coherent detection set-up, and the signals were captured with four photo-detectors using 
an $80 \mathrm{GSa} / \mathrm{s}, 25 \mathrm{GHz}$ bandwidth oscilloscope for analogue to digital conversion. DSP was used offline with standard algorithms for signal recovery and linear transmission impairments compensation. Q factors were calculated from bit-wise error counting, and averaged over two million bits.

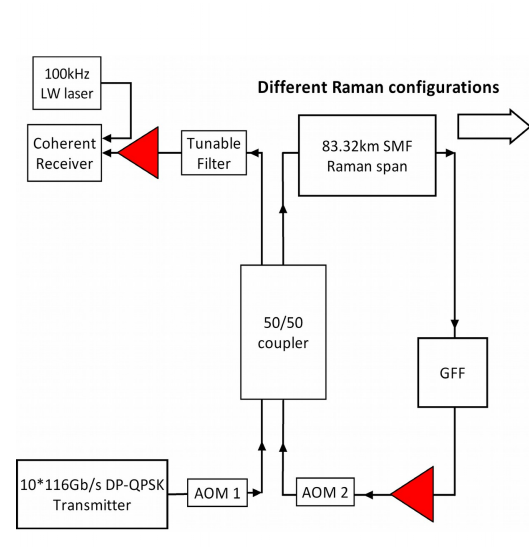

a

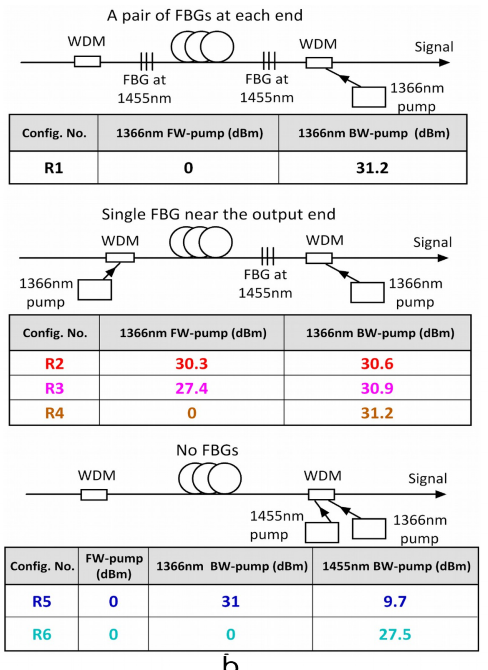

b

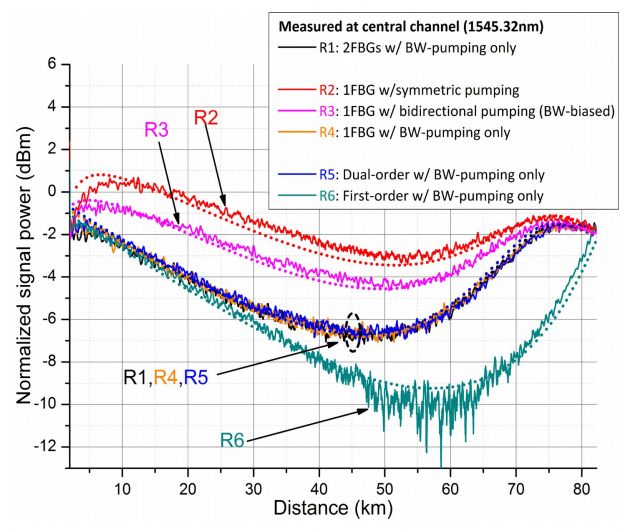

Fig. 1.(a). Experimental set-up of transmitter, recirculating loop, and coherent receiver; (b). Schematic diagram of tested Raman configurations; (c). Simulations (dot line) and experimental data (solid line) of SPVs using different Raman configurations.

As a baseline, second-order $1366 \mathrm{~nm}$ backward pumping configuration (R1) with a Fabry-Perot cavity (FBG at each end of the span) was used. Previous experiments have shown this to give improved performance compared with lumped amplification with EDFAs [2]. Schematic diagrams and pump powers for the Raman configurations tested are shown in Fig. 1.(b). For all configurations the Raman gain was set to counterbalance the $16.5 \mathrm{~dB}$ attenuation of the fiber. The FBGs used were centered at $1455 \mathrm{~nm}$ with $0.5 \mathrm{~nm} 3 \mathrm{~dB}$ bandwidth and $95 \%$ reflectivity. In the random DFB fiber laser based amplifiers (R2, R3 \& R4), a single FBG was used at the output end of the span. The first-order random laser at $1455 \mathrm{~nm}$ was generated by the resonant mode reaching the lasing threshold in a distributed cavity formed by a distributed feedback (Rayleigh scattering) and an FBG [6,7]. Three pump power combinations were used in this configuration, as forward pumping at $1366 \mathrm{~nm}$ could amplify the signal from the input section of the fiber by amplifying the forward-propagated random lasing. For comparison backward first-order and dual-order pumping with no FBGs (R5 \& R6) were also tested. For all configurations the $1366 \mathrm{~nm}$ second-order and $1455 \mathrm{~nm}$ first-order pumps were highly depolarized and coupled into the span through WDM couplers.

SPVs along the transmission span measured using a modified optical time-domain reflectometer (OTDR) are shown in Fig. 1.(c) and confirmed with simulations (dotted lines) [1].

The baseline measurement (R1) had an SPV of $\sim 5 \mathrm{~dB}$. Using a bi-directionally pumped random DFB fiber laser, the SPVs were reduced to $3.6 \mathrm{~dB}$ in configurations R2 (symmetrical pumping) \& R3 (BW-biased pumping). The performance of backward only pumped random laser based scheme (R4) was identical to the baseline Fabry-Perot backward only pumping (R1). This indicates, for this configuration the FBG at the input end of the span didn't actually contribute to the amplification, and the FBG at the input end for R1 was superfluous.

With no FBG (R5), backward dual-order pumping (1366 nm and $1455 \mathrm{~nm})$ can be used to give the same SPV as $\mathrm{R} 1$ and R4 with similar second-order pump power and relatively low first-order pump power $(\sim 10 \mathrm{dBm})$. This did however require two pump wavelengths and careful control of first-order pump power, which makes the simplicity of R4 attractive $[1,8]$. Configuration R6 used only backward first-order pumping and gave the highest SPV of $\sim 9 \mathrm{~dB}$.

\section{Transmission results and discussions}

Fig. 2.(a) shows $\mathrm{Q}$ factors versus transmission distances and 2.(b) shows $\mathrm{Q}$ factors versus launch power sweeps at $3333 \mathrm{~km}$ for all Raman configurations. The random laser scheme R3 (bidirectional pumping biased towards backward pump) gave the longest transmission distance of $7915 \mathrm{~km}$. As expected from SPVs shown in Fig. 1.(c), the impact of nonlinearities in R2 (bidirectional symmetric pumping) degraded transmission performance - the SPVs of R3 and R2 were the same (3.6 dB) but R2 had higher average signal power. This led to a lower optimum launch power and a reduced maximum transmission distance of $7082 \mathrm{~km}$. The random laser scheme R4 (backward pumping only) had a higher SPV value of $5 \mathrm{~dB}$ which led to a higher optimum launch power and a reduction in reach to 7082 km. As seen in Fig. 1.(c), the second FBG used in R1 didn't contribute to the amplification, so it had the same 
performance as R4. Dual-order pumping without FBG (R5) also gave similar performance due to the same SPV as $\mathrm{R} 1$ and R4. With first-order backward pumping (R6), the optimum launch power of $-5 \mathrm{dBm}$ was achieved, and the transmission distance was decreased to $4999 \mathrm{~km}$. As shown in Fig. 2.(b), nonlinear threshold changes as the integral of SPV because effective nonlinear length increases. Fig. 3 shows OSNR, Q, and spectra at maximum transmission distances for the three random laser schemes R2-R4 and as comparison R6 (first-order backward pumped). All measured channels were below the FEC threshold corresponding to $3.8 \times 10^{-3}$ in bit error rate.

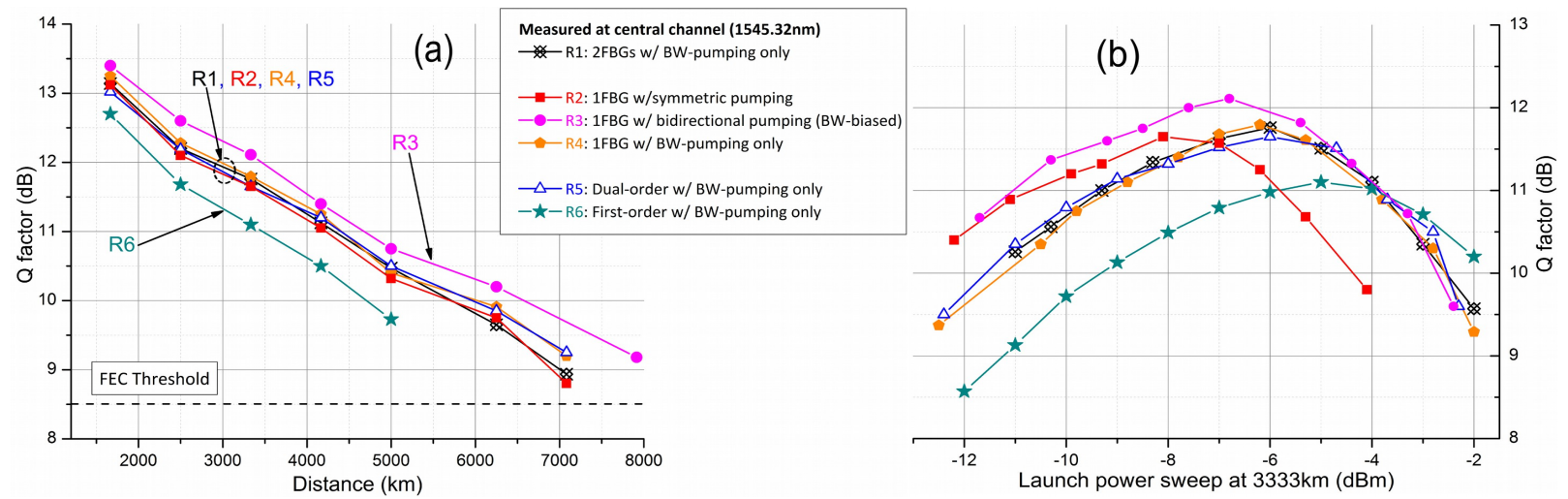

Fig. 2.(a) $Q$ factors versus transmission distances; (b) $Q$ factors versus launch power per channel at $3333 \mathrm{~km}$
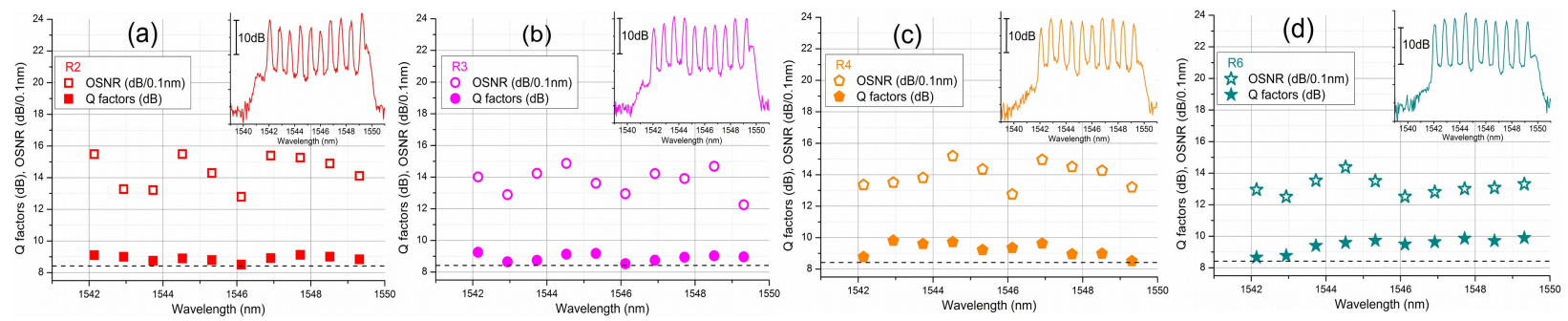

Fig. 3. OSNRs, Q factors, and received spectra measured at its maximum reach;

(a). Bi-directionally pumped random laser scheme R2 (symmetric pumping) at $7082 \mathrm{~km}$; (b). Bi-directionally pumped random laser scheme R3 (BW-biased pumping) at 7915km; (c).BW-pumped random laser scheme R4 at 7082km; (d). BW-pumped first-order scheme R6 at $4999 \mathrm{~km}$.

\section{Conclusion}

We have presented a detailed investigation of long haul 116G DP-QPSK coherent transmission using different Raman amplification techniques. The best performance $(7915 \mathrm{~km})$ was achieved with a random DFB fiber laser based configuration which included bi-directionally second-order pumping. In summary, we have shown that this scheme offers the best transmission performance whilst maintaining a low signal power variation (low effective noise figure and consequently better OSNR), and as such the scheme is also highly suitable for different techniques of nonlinearity compensation $[2,3]$.

\section{Acknowledgement}

This work was funded by UK EPSRC Programme Grant UNLOC EP/J017582/1. The Authors thank Changle Wang and Lin Zhang for providing FBGs, and thank Juan. D. Ania-Castañón and Dmitry Churkin for useful discussions.

\section{References}

[1] J. D. Ania-Castañón, "Quasi-lossless transmission using second-order Raman amplification and fiber Bragg gratings,” Opt. Express 12, 43724377 (2004).

[2] I. D. Phillips et al., "Exceeding the nonlinear Shannon Limit using Raman Laser Based Amplification and Optical Phase Conjugation," in Optical Fiber Communication Conference(Optical Society of America, San Francisco, California, 2014), p. M3C.1.

[3] J. E. Prilepsky et al., "Nonlinear Inverse Synthesis and Eigenvalue Division Multiplexing in Optical Fiber Channels," Phys. Rev. Lett. 113, $0139011-5,2014$.

[4] M. Tan et al., "Long-haul Transmission Performance Evaluation of Ultra-long Raman Fiber Laser Based Amplification Influenced by Second Order Co-pumping," in Asia Communications and Photonics Conference(Shanghai, China, 2014), ATh1E.4.

[5] X. Jia et al., "Random-lasing-based distributed fiber-optic amplification," Opt. Express 21, 6572-6577 (2013).

[6] S. K. Turitsyn et al., "Random distributed feedback fiber lasers," Physics Reports 542, 133-193 (2014).

[7] W. L. Zhang et al., "Low threshold $2^{\text {nd }}$-order random lasing of a fiber laser with a half-opened cavity," Opt. Express 20, 14400-14405 (2012).

[8] J.-C. Bouteiller et al., "Dual-order Raman pump providing improved noise figure and large gain bandwidth," in Optical Fiber Communication Conference(Optical Society of America,2002), p. FB3. 